\title{
AGE DIFFERENCES IN DRIVING-SPECIFIC TESTS OF EXECUTIVE FUNCTION
}

\author{
Ann E. Lambert, Daniel J. Cox, Melissa L. O’Connor, Rick L. Moncrief, \\ Clarissa Cho \& Ronald J. Johnson \\ University of Virginia Health System \\ Charlottesville, Virginia, USA \\ Email: ann.lambert@virginia.edu
}

\begin{abstract}
Summary: The purpose of the present study was to examine age differences in executive function as measured by novel driving-specific tests of executive function using a novel driving simulator. Developmental changes in executive function have been implicated as possible contributing factors to elevated crash statistics for both older adult (over age 65) and adolescent (between age 15 and 20) populations, however for different reasons. Poorer older adult driving performance has been partially attributed to general age-related cognitive decline in executive function mediated by age-related frontal-lobe atrophy and neural disconnection. Immature executive function has been implicated in poorer adolescent driving performance and is thought to be expressed in situations where the developmentally high sensitivity of the socio-emotional reward system outcompetes the regulatory influence of the under developed executive system. Using a new, high fidelity, virtual reality driving simulator, we created drivingspecific tests to assess executive function. These operational tests employed driving-relevant stimuli, with driving-relevant challenges, that required drivingrelevant responses, in a driving-relevant context. Fifteen older adult and 20 adolescent drivers completed these driving-specific executive function tests. We hypothesized that poorer older adult driving performance would be reflected on these driving specific tests of executive function due to general cognitive decline and that, given the absence of social-emotional reward, adolescents would outperform older adults. Analyses of both bivariate correlations and group comparisons generally supported these predictions.
\end{abstract}

\section{BACKGROUND}

\section{Older Adults and Adolescents: Two Populations at High Risk for Traffic Fatalities}

Older adult (over the age 65 years) and adolescent (between the ages of 15 and 20 years) drivers are two populations at high risk of traffic-related accidents and fatalities. In 2008, older adult drivers represented $15 \%$ of the driving population but accounted for $15 \%$ of all traffic fatalities, $14 \%$ of all vehicle occupant fatalities and $18 \%$ of all pedestrian fatalities (National Highway and Transportation Safety Administration: NHTSA, 2008a). Older adults are also more likely to be considered at-fault in a collision (Langford, Koppel, Andrea, \& Fildes, 2006). Statistics are similarly concerning for adolescent drivers. Motor vehicle accidents are the leading cause of death for adolescents in the United States (Center for Disease Control and Prevention, 2012) and, in 2008 , adolescent drivers represented $6.4 \%$ of the driving population but accounted for $12 \%$ of all fatal crashes and 14\% of all police-reported crashes (NHTSA, 2008b). 


\section{Executive Function and Driving Performance}

For both these populations, developmental changes in executive function have been implicated as possible factors contributing to their high-risk status (Anstey, Wood, Lord, \& Walker, 2005; Gardner \& Steinberg, 2005). Cognitive psychologists and Neuropsychologists use the term executive function to refer to a constellation of cognitive processes, mediated by prefrontal brain regions, which facilitate control of our thoughts and actions (Kane \& Engle, 2002). These processes include response inhibition, the ability to refrain from a habitual or over-learned response, and working memory, the ability to maintain information in the face of distraction. The frontal lobes of the brain are the slowest to develop, not reaching maturity until 25 to 30 years of age. They are also the earliest areas to decline with normal cognitive aging, a process that typically begins in the $30^{\text {th }}$ decade of life (Watson, Lambert, Miller, \& Strayer, 2011). Thus, across the lifespan, the developmental trajectory of the frontal lobes and cognitive processes they support form an inverted u-shaped curve. Interestingly, when traffic-related fatalities are plotted across the lifespan they form a mirror image with traffic-related fatalities being high during adolescence, gradually decreasing until middle age, and remaining stable until older adulthood when they rise exponentially (Watson, Lambert, Miller, \& Strayer, 2011).

While executive function is one factor possibly contributing to traffic fatality statistics for both older adult and adolescent drivers, the way in which declining executive function impacts older drivers and immature executive function impacts adolescent drivers has been conceptualized in different ways. On one hand, the older adult statistics have been partially attributed to age-related frontal area atrophy and neural disconnection which leads to declines in executive functions (Park \& Gutchess, 2000). As these cognitive deficits become increasingly severe, they may begin to affect driving competence. On the other hand, adolescent traffic statistics have been partially attributed to the developmental interplay between two cognitive systems and how this interplay may, in certain situations, lead to risky driving (Gardner \& Steinberg, 2005). This dual-systems model postulates that adolescent risk-taking behind the wheel results from an imbalance in the development of the executive system, mediated by frontal areas of the brain and the socioemotional reward system, mediated by limbic regions of the brain. During adolescence, the executive system develops gradually whereas the sensitivity of the socio-emotional reward system peaks (Gardner \& Steinberg, 2005). It is postulated that the socio-emotional reward system may drown out the regulatory influence of the relatively immature executive system in situations where reward salience is high, such as driving in the presence of peers (Gardner \& Steinberg, 2005). However, in the absence of high reward salience, adolescent executive function is likely sufficiently mature to tackle the attentional demands of driving.

Thus, if age-related declines in executive functions are responsible for poorer older adult driving performance, this should be reflected on driving specific tests of executive function. If underdeveloped executive function is responsible for poorer adolescent driving performance when socio-emotional reward salience is high, then, in the absence of social-emotional reward, adolescents should outperform older adults on driving specific tests of executive function. 


\section{The Model T3 Driving Simulator}

Using an ATARI Research Driving Simulator, our group previously demonstrated that performance in the simulator predicted future collisions (Cox, Taylor, \& Kovatchev, 1999). Thus, driving simulation has significant utility regarding the safe and objective assessment of driving competence. While the ATARI Research Driving Simulator served as a reliable research instrument for two decades, driving simulation technology has significantly advanced since its development. Consequently, we have collaborated with programmers and engineers across the country to build a new, high fidelity, virtual reality (VR) driving simulator we refer to as the Model T3 simulator (see photo). A unique element of our Model T3 driving simulation system is the development of driving-specific operational tests. Through extensive consultation with driving experts (neuropsychologists, cognitive

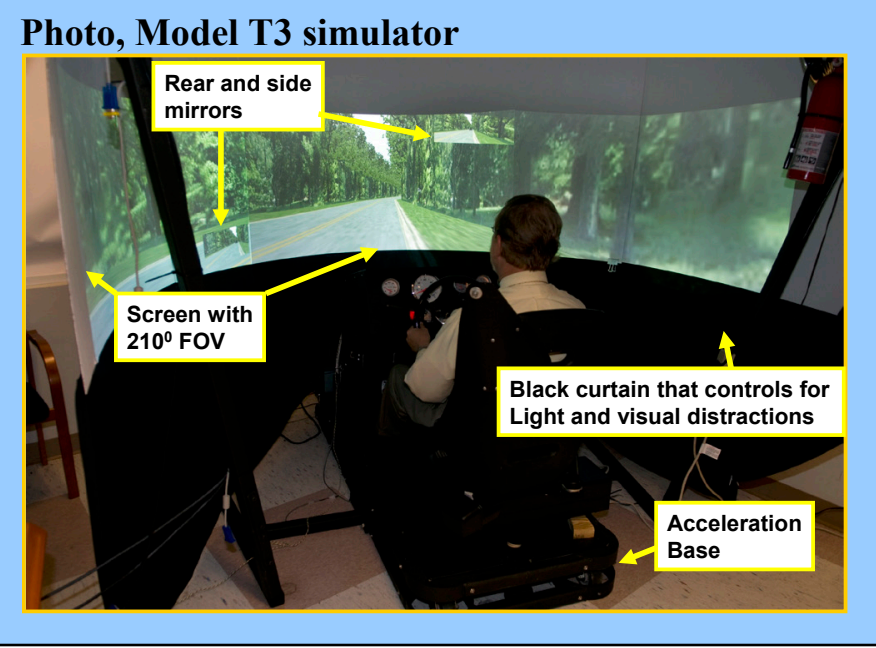
psychologists, clinical psychologists, and engineers) we created a subset of these tests to assess executive function using driving-relevant stimuli, with driving-relevant challenges, requiring driving-relevant responses, in a driving-relevant context.

\section{OBJECTIVES}

The present study aimed to a) examine relationships between age and performance on new driving-specific tests of executive function administered within the Model T3 virtual reality driving simulator and to b) examine age differences in performance on these tests using two high risk groups of drivers: older adults and adolescents. We predicted that age would be negatively associated with driving-specific executive function abilities, positively associated with drivingspecific executive function errors, and that older adults would make more errors due to agerelated declines in executive function.

\section{METHODS}

\section{Participants}

Fifteen older adult and 20 adolescent driver about to obtain their independent driver's licenses, participated in the present study. All older drivers ethnicity was White/Caucasian. Eight older adult drivers were female. The older adult sample averaged 74.93 years of age and had completed an average of 16.73 years of education. Of the adolescent sample, three participants' ethnicity was African American, two were Hispanic, two preferred not to respond and the remaining were White/Caucasian. Eight of the adolescents were female. The adolescent sample averaged 16.10 years of age and had completed an average of 10.45 years of education. 


\section{Apparatus}

This study used the Model T3 driving simulator. The simulator displayed a $210^{\circ}$ field of view on a white curved screen inside an 8 foot cylinder. The visual driving environment was generated by three digital projectors, each projecting $70^{\circ}$ of the visual image. Included in the projection were side and rear view mirrors. The simulator included optional automatic and manual transmission with reverse, seatbelt, dashboard, steering wheel, turn signal, gas and brake controls, air conditioning, and a fixed seat with a sliding console to control the driver's position relative to the projected image. To simulate movement, the quadraphonic sound system incorporated Doppler sound presentation that portrayed engine, tire and traffic noises. Steering incorporated forced feedback to simulate road texture and tire friction. All assessments were delivered by our driving simulation software.

\section{Design}

Our design was correlational and quasi-experimental. We aimed to investigate the bivariate relationships between age (our independent variable) and driving-specific executive function indices collected via our Model T3 driving simulator. These indices comprised our dependent variables and included 1) percentages of braking inhibition and steering inhibition errors, 2) percentages of unsafe driving divided attention errors, and 3) driving-specific working memory capacity. We were also interested in group differences (older adult vs. adolescent drivers) in performance on the driving specific executive function dependent variables listed above.

\section{Procedure}

Response contingency training. All participants first gave informed consent prior to completing the driving scenarios. The first scenario required drivers to process two driving instruction goals simultaneously in order to train a response contingency. In this scenario, the driver followed a lead vehicle at a fixed speed, distance, and lane position. The lead vehicle's brake lights came on periodically for short 0.5 second durations and long 3.0 second durations. The drivers were instructed to remove their foot from the accelerator and press the brake as soon as the brake lights were detected. In addition to the braking task, the drivers engaged in a steering task. Periodically, the lead vehicle's rear wheels passed over "filled" potholes that were gray in color and "deep, unfilled" potholes that were black in color. The drivers were instructed to avoid both filled and deep potholes by steering around the potholes without leaving their lane. During the course of this operational test, drivers developed a response contingency to brake for all brake lights and to swerve for all potholes. The primary purpose of this test was to develop response contingencies critical to the dependent variables later collected in the response inhibition and working memory operational tests.

Response inhibition operational test. The second scenario was an executive function operational test, the response inhibition test, which tapped the executive function of inhibition by requiring drivers to inhibit 2 of the 4 previously trained response contingencies. As in the response contingency training, drivers again followed a lead vehicle at a fixed speed, distance, and lane position. As before, the lead vehicle's brake lights came on periodically for short 0.5 second durations and long 3.0 second durations. Again, the drivers were instructed to remove their foot 
from the accelerator as soon as the brake lights were detected however, this time, they were instructed not to press the brake when the lead vehicle's brake lights came on for a short duration and to only press the brake if the lights came on for a long duration. In this way, drivers were required to inhibit the previously trained response contingency to brake to all brake lights and to discriminate between long brake lights that would require braking behavior and short brake lights that would not require braking. As in the response contingency training, the drivers also engaged in a steering task. As before, periodically, the lead vehicle's rear wheels passed over "filled" potholes that were gray in color and "deep, unfilled" potholes that were black in color. This time, the drivers were instructed to avoid only the dangerous deep potholes by steering around them without leaving their lane. They were to ignore the safe filled potholes by refraining from steering around them. In this way, drivers were required to inhibit the previously trained response contingency to steer around all potholes and to discriminate between safe filled potholes and dangerous deep potholes. Our dependent variables of interest in this test were percentage braking inhibition errors (braking in response to a short brake light), percentage steering inhibition errors (steering in response to a filled pothole), and percentage unsafe driving divided attention errors (hitting a deep unfilled pothole).

Working memory operational test. The final executive function operational test was the working memory operational test which built upon the response inhibition test by requiring participant to maintain in working memory the identity of street signs while also adhering to response inhibition instructions from the preceding test. In most respects, this test was identical to the response inhibition operational test with drivers again following a lead vehicle at a fixed speed, distance, and lane position while also braking to short brake lights and swerving around deep potholes. However, in this test they were told that they would be passing common road signs and they were asked to try to remember these signs for a later test. After passing a series of signs that ranged from 1 to 3 signs in number, the car would automatically come to a complete stop and a sign recognition array would be displayed in the virtual environment. Drivers were asked to select the signs they had passed since the last recognition array in the same serial order that they were passed. Our dependent variables of interest in this test were again, percentage braking inhibition errors, percentage steering inhibition errors, and percentage unsafe driving divided attention errors. We were also interested in driving working memory capacity which was determined based on an absolute system used to score complex span tasks (Conway et al., 2005) This was done by awarding one point for each sign recalled in the correct serial order only for sets of signs wherein all signs were recalled correctly in the correct serial order.

\section{RESULTS}

Pearson bivariate correlations examined relationships between the independent variable of age and the dependent driving variables of error percentages in response inhibition braking inhibition, response inhibition steering inhibition, response inhibition divided attention, working memory braking inhibition errors, working memory steering inhibition errors, working memory divided attention as well as the driving-specific executive function ability of driving working memory capacity. Please see the Table below for a complete correlation matrix. 
Table 1. Bivariate correlations between age and dependent driving variables

\begin{tabular}{|c|c|c|c|c|c|c|c|}
\hline Measure & 1 & 2 & 3 & 4 & 5 & 6 & 7 \\
\hline 1. Age & - & & & & & & \\
\hline 2. Response inhibition braking inhibition errors & .29 & - & & & & & \\
\hline 3. Response inhibition steering inhibition errors & .10 & -.03 & - & & & & \\
\hline 4. Response inhibition divided attention errors & $.64 *$ & 0.18 & -.22 & - & & & \\
\hline 5. Working memory braking inhibition errors & -.02 & $.36^{*}$ & .09 & -.28 & - & & \\
\hline 6. Working memory steering inhibition errors & .14 & .25 & .32 & -.11 & .19 & - & \\
\hline 7. Working memory divided attention errors & $.62 *$ & .14 & -.19 & $.77 *$ & -.12 & -.24 & - \\
\hline 8. Driving working memory capacity & $-.59 *$ & -.21 & .13 & $-.44 *$ & .01 & -.09 & $-.48 *$ \\
\hline
\end{tabular}

As predicted, age was negatively associated with driving-specific executive function ability. Specifically, age was strongly negatively correlated with driving working memory capacity $(r=$ $-.59, p<.001)$. Also, age and some driving specific executive function error percentages were positively associated. Specifically, age was strongly associated with response inhibition divided attention error probability $(r=.64, p<.001)$ and strongly associated with working memory divided attention error probability $(r=.62, p<.001)$. However, counter to prediction, age did not significantly correlate with braking $(\mathrm{r}=.29, \mathrm{p}=.087)$ and steering inhibition $(\mathrm{r}=.10, \mathrm{p}=$ $.974)$ error probability in the response inhibition test or with braking $(r-.02, p=.916)$ and steering inhibition $(\mathrm{r}=.14, \mathrm{p}=.412)$ error probability in the working memory test.

To better understand the nature of these age differences, Students t-tests were used to examine group differences in driving-specific executive function ability and driving-specific executive function errors. Statistically significant $(p<.05)$ group differences were found between older adult and adolescent drivers in driving working memory capacity, $t(33)=3.55, p=.001$, response inhibition divided attention error percentages $t(33)=-4.45, p<.001$, and working memory divided attention error percentages $t(33)=-4.33, p<.001$. Older adults had lower driving working memory capacity and had a higher percentage of response inhibition divided attention error percentages on both the response inhibition and working memory tests than did adolescents. Group differences between braking and steering inhibition error percentages on the response inhibition test and the working memory test failed to reach significance (all $p \mathrm{~s}>.05$ ).

\section{CONCLUSION}

This study examined relationships between age and driving performance and group differences between older adult and adolescent drivers on new driving-specific tests of executive function. Correlational and group comparisons were consistent with predictions for driving working memory capacity and divided attention error percentages in both the response inhibition and the working memory tests. However, the expected bivariate relationships and group differences were not observed between braking and steering inhibition error percentages for either the response inhibition or working memory tests. This absence of age differences in inhibition error percentages suggests that both older adults and adolescents are equally able to inhibit a previously learned driving-relevant response contingency; however, for older adults, this inhibition entails a greater divided attention cost. When under an inhibitory load, older adults hit a greater number of dangerous deep potholes than adolescents. These results shed light on age differences in driving-specific executive function and support the validity of the Model T3 
Driving Simulator's executive function tests. Future research examining driving specific executive function in adolescent drivers alone vs. in the presence of peers could help to support the simulator's utility in assessing the impact of immature executive function in teen drivers.

\section{ACKNOWLEDGEMENTS}

SBIR Phase I and Phase II support for this new clinical tool was provided by DARPA. MBFARR directed and produced the systems used for this research. More information on the product can be found at www.generalsimulation.com.

\section{REFERENCES}

Anstey, K. J., Wood, J., Lord, S., \& Walker, J. G. (2005). Cognitive, sensory and physical factors enabling driving safety in older adults. Clinical Psychology Review, 25(1), 45-65.

Centers for Disease Control and Prevention. (2012). Injury prevention \& control: motor vehicle safety teen drivers fact sheet. Retrieved from http://www.cdc.gov/motorvehiclesafety/teen drivers/teendrivers factsheet.html

Cox, D. J., Taylor, P., \& Kovatchev, B. (1999). Driving simulation performance predicts future accidents among older drivers. Journal of the American Geriatric Society, 47, 381-382.

Gardner, M., \& Steinberg, L. (2005). Peer Influence on Risk Taking, Risk Preference, and Risky Decision Making in Adolescence and Adulthood: An Experimental Study. Developmental Psychology, 41(4), 625-635.

Kane, M. J., \& Engle, R. W. (2002). The role of prefrontal cortex in working-memory capacity, executive attention, and general fluid intelligence: An individual-differences perspective. Psychonomic Bulletin \& Review, 9(4), 637-671.

Langford, J., Koppel, S., Andrea, D., \& Fildes, B. (2006). Determining older driver crash responsibility from police and insurance data. Traffic Injury Prevention, 7(4), 343-351.

National Highway and Transportation Safety Administration. (2008a). Traffic safety facts 2008 data: older population. (NHTSA's National Center for Statistics and Analysis). Retrieved from http://www-nrd.nhtsa.dot.gov/Pubs/811161.pdf

National Highway and Transportation Safety Administration. (2008b). Traffic safety facts 2008 data: young drivers. (NHTSA's National Center for Statistics and Analysis). Retrieved from http://www-nrd.nhtsa.dot.gov/Pubs/811169.pdf

Park, D. C., \& Gutchess, A. (2000). Cognitive aging and everyday life. In D. C. Park, N. Schwarz (Eds.), Cognitive aging: A primer (pp. 217-232). New York, NY US: PsychologyPress.

Watson, J. M., Lambert, A. E., Miller, A., \& Strayer, D. L. (2011). The magical letters P, F, C and sometimes $\mathrm{U}$ : The rise and fall of executive attention with the development of prefrontal cortex. In K. L. Fingerman, C. A. Berg, J. Smith, T. C. Antonucci (Eds.), Handbook of lifespan development (pp. 407-436). New York, NY US: Springer Publishing Co. 